Canad. Math. Bull. Vol. 29 (4), 1986

\title{
FUZZY SET THEORY AND TOPOS THEORY
}

BY

MICHAEL BARR

\begin{abstract}
The relation between the categories of Fuzzy Sets and that of Sheaves is explored and the precise connection between them is explicated. In particular, it is shown that if the notion of fuzzy sets is further fuzzified by making equality (as well as membership) fuzzy, the resultant categories are indeed toposes.
\end{abstract}

1. Introduction. A fuzzy set is a map $\xi: X \rightarrow[0,1]$. If $\eta: Y \rightarrow[0,1]$ is another fuzzy set, a morphism is defined, e.g. in Goguen, [5] to be a function $f: X \rightarrow Y$ such that $\xi \leq f \circ n$. This definition is justified in various ways, but the simplest is that it is suggested by the definitions of intersection and unions of fuzzy sets.

In trying to figure out some of the consequences of this definition, I quickly came to the realization that the theory of fuzzy sets is deficient in one respect: fuzzy sets are not fuzzy enough!

The reason is very simple. Set theory is built out of two predicates: membership and equality. For example, two sets $X$ and $Y$ are equal iff $(x \in X) \Leftrightarrow(y \in Y)$. In the theory of fuzzy sets one of the two predicates, membership, is made to take values in the unit interval and thus become fuzzy while the other is left crisp. This results in a certain incoherence in the theory. The most obvious example of this is the lack of any analogue of power sets (the set of all subsets, by which one would mean the set of all fuzzy subsets). The reason for this is not hard to find. If two subsets have all the same elements below a certain level $t$, then one would expect that they are equal below that level. But there is no way to express that fact in the language of fuzzy set theory.

One way to repair this would be the following. Take a fuzzy set to be a pair $(\xi: X \rightarrow$ $[0,1], \epsilon: E \rightarrow[0,1])$ such that $E$ is an equivalence relation on $X$. By this I mean that $E \subseteq X \cdot X$ is an equivalence relation in the usual sense and that the two projection maps $E \rightrightarrows X$ are morphisms in the same category. This means that if $(x, y) \in E$ with $\epsilon(x, y)=t$ while $\xi(x)=r$ and $\xi(y)=s$, then both $t \leq r$ and $t \leq s$. The interpretation of this is as follows: the degree of membership of $x$ is $r$ and that of $y$ is $s$, but the degree to which $x=y$ is $t$. It is, in fact, perfectly possible to carry out this idea in detail and use it to get a theory in which both membership and equality are fuzzy and in which all logical operators (including higher order) are definable.

Received by the editors May 13, 1985, and, in revised form, July 19, 1985.

AMS Subject Classification (1980): Primary 18B25, 03E72; Secondary 03G30.

(C) Canadian Mathematical Society 1985. 
But it is awkward. For example, to define a morphism from $(\xi: X \rightarrow[0,1]$, $\epsilon: E \rightarrow[0,1])$ to $(\eta: Y \rightarrow[0,1], \phi: F \rightarrow[0,1])$, you have to take a relation between $X$ and $Y$ and impose a functionality condition in terms of $E$ and $F$. Fortunately, there is a better way. Category theorists will recognize that what $I$ have done is to force equivalence relations to be effective. To explain: equivalence relations can be defined in arbitrary categories. In some, but not all, categories it is possible to form a quotient modulo arbitrary equivalence relations. Such categories are said to have effective equivalence relations. Under certain conditions, called regularity, it is possible to embed a category in a category with effective equivalence relations in a standard way. When this is done for the category of fuzzy sets, the resultant category turns out to be a well known category: the category of sheaves on a locale (defined below). It follows that well known constructions like power sets and exponentials (function sets) are readily definable and that all constructions of intuitionistic set theory can be carried out.

There is another problem with Goguen's definition. If we think of the function $\xi: X \rightarrow[0,1]$ as assigning to each element a degree of membership in $X$, then it would seem that the elements $x$ with $\xi(x)=0$ aren't there at all. But Goguen's morphisms are required to be defined there and two such morphisms that agree except on elements of degree of membership 0 are not equal. One result of this is that the lattice of subobjects of the fuzzy set $\xi: X \rightarrow[0,1]$ consists not only of functions on $X$ that are subordinate to $\xi$, but also of such functions defined on subsets of $X$. In particular, we can easily see that the lattice of subobjects of the one element set is not $[0,1]$, but the lattice we will call below $[0,1]^{+}$, the lattice with a new initial element adjoined. This is the reason for the odd condition, going under the name disjointedly c.d.l.ordered, that appears in Goguen's formulation. It turns out that if that condition is repaired, the definition of morphism has to be more complicated. Since the moral of this paper is that the correct generality is that of a topos (in which these difficulties disappear), we will keep this condition unchanged for as long as we are analyzing Goguen's category.

Of course, all this works in the generality of a locale, that is a complete lattice in which finite infs distribute over all sups. They are otherwise known as Heyting algebras, but morphisms of locales are required to preserve only the sups and the finite infs. Goguen restricted himself, for unknown reasons, to lattices that are both locales and colocales. One reason that the generality considered here is interesting is that the open set lattice of a topological space is a locale (hence the name) and rarely a colocale.

Our main result is that Goguen's category does not form a topos (with the sole exception being the case that the lattice is the one element set), but that if one imposes the condition that equivalence relations be effective, the resultant category is a topos. In fact the toposes that arise from Goguen's construction can be characterized as the toposes of the form $\operatorname{Sh}(L)$ where $L$ is a locale that has a smallest non-zero element.

Another writer has written on these subjects, this time claiming that fuzzy sets do form a topos (Eytan, [3]). He uses a different definition of morphism, with the result that his categories do form a topos when $L$ is a Boolean algebra (Pitts, [8]), but not 
otherwise. The general claim was shown false by Pitts. Essentially, his construction repairs the second problem alluded to above (concerning the elements of degree of membership 0), but does not solve the problem of effective equivalence relations. The problem appears to be in his Proposition 2.4.2 that claims toposhood for a certain class of categories and for which not the least hint of proof is offered. Further errors in that paper include the claim that Goguen's category is a subcategory of the category of fuzzy sets constructed there (it isn't because of the fact mentioned above that for Goguen two morphisms that agree except on some elements of membership degree 0 are different), the claim that this inclusion has a right adjoint and the systematic misspelling of the name Kronecker. However the most egregious error was the failure to appreciate the fact that Goguen's axiom of disjointed c.d.1.-ordering was a conclusion, not a hypothesis imposed by Goguen; and that if it wasn't satisfied in the category constructed by Eytan, then something was radically wrong. Had this clue been followed in the proper spirit of scientific inquiry, then the whole web of errors in Eytan's paper would have unravelled.

2. The categories $\operatorname{Fuz}(L), \operatorname{Sh}(L)$ and $\operatorname{Mon}(L)$. Let $L$ be a locale. Following Goguen, we denote by $\mathbf{F u z}(L)$ the category whose objects are functions $\xi: X \rightarrow L$. A morphism $f:(\xi: X \rightarrow L) \rightarrow(\eta: Y \rightarrow L)$ is a morphism $f: X \rightarrow Y$ such that $\xi \leq f \circ \eta$.

If $\xi: X \rightarrow L$ is an object of $\mathbf{F u z}(L)$, there is another way of thinking of it. For $a \in L$, let $X(a)$ denote $\xi^{-1}(a) \subseteq X$. Then $X$ is the disjoint sum, denoted $\Sigma_{a \in L} x(a)$ of these subsets. In fact an object of Fuz $(L)$ can be thought of as an $L$ indexed family of sets. Then $X(a)$ can be thought of as the set of all elements whose degree of membership in $X$ is just $a$.

Let $L$ be a locale. We describe a category called the category of $L$-valued sets and denoted $\operatorname{Sh}(L)$ (sheaves on $L$ ). An object is an $L$ indexed family of sets $\{S(a) \mid a \in L\}$ together with a function $S(b) \rightarrow S(a)$ whenever $a \leq b$, denoted $x \mapsto x \mid a$, for $x \in S(b)$. This is called a presheaf provided that whenever $a \leq b \leq c$, and $x \in s(c)$, $x \mid c=x$ and $(x \mid b)|a=x| a$ and is called a sheaf if it satisfies one additional condition (the sheaf condition):

Suppose $a=V_{i \in I} a_{i}$ and for each $i \in I$ we have an element $x_{i} \in S\left(a_{i}\right)$ such that for all $i, j \in I, x_{i}\left|a_{i} \wedge a_{j}=x_{j}\right| a_{i} \wedge a_{j}$. Then there must exist a unique element $x \in S(a)$ such that $x \mid a_{i}=x_{i}$.

In categorical language, this condition amounts to the hypothesis that $S(a) \rightarrow$ $\pi S\left(a_{i}\right) \rightrightarrows S\left(a_{i} \wedge a_{j}\right)$ is an equalizer.

We note that since in a lattice the empty set covers the bottom element and, since an empty product is defined to be a one element set, it follows that for any sheaf $S$, $S(0)=1$. Furthermore the restriction to 0 evidently identifies every element. This should not be too surprising since it reflects the fact that false implies everything. We define a full subcategory Mon $(L) \subseteq \mathbf{S h}(L)$ which consists of all sheaves $S$ for which $0<a \leq b$ implies $S(b) \rightarrow S(a)$ is a $1-1$ function. Thus all restrictions are monic, except the restriction to 0 . 
A morphism $f: S \rightarrow S^{\prime}$ is an $L$ indexed family of functions $f(a): S(a) \rightarrow S^{\prime}(a)$ such that for $a \leq b$, and $x \in S(b), f(x) \mid a=f(x \mid a)$.

All three of these categories $\mathbf{F u z}(L), \mathbf{S h}(L)$ and $\mathbf{M o n}(L)$ appear naturally as $L$ indexed families of sets. However, the first consists at $a \in L$ of those elements whose degree of membership is exactly $a$, while the other two consist at $a$ of those whose degree of membership is at least $a$. That is why there are restriction maps. The possible failure of these maps to be injective in the case of $\mathbf{S h}(L)$ is what allows two elements to be equal at some lesser stage than the stage at which they first exist.

Let $L$ be a locale and suppose $z$ is an element not in $L$. Let $L^{+}$denote the locale with elements $L \cup\{z\}$ in which $z$ precedes every element of $L$. In other words, it is $L$ with a new initial element. We can now briefly describe our main results. The first is that the category $\mathbf{F u z}(L)$ is equivalent to Mon $\left(L^{+}\right)$and the second is that $\mathbf{S h}\left(L^{+}\right)$is the effective completion of either of them.

3. Categorical properties. The locales of the form $L^{+}$are special and some of our results below are not valid in general. However the only special property they depend on is that the inf of two non-zero elements is non-zero. Any chain, for example, has this property. Let us say that a locale is totally connected if the inf of two non-zero elements is non-zero. The reason for the name is that the condition means that every element of the locale is connected.

Proposition 1. Let $L$ be totally connected. Then Mon $(L) \subseteq \mathbf{S h}(L)$ has the following properties:

(i) Every subobject in $\mathbf{S h}(L)$ of an object of $\operatorname{Mon}(L)$ belongs to $\operatorname{Mon}(L)$.

(ii) Every product of objects of Mon $(L)$ belongs to Mon $(L)$.

(iii) Every object of $\mathbf{S h}(L)$ is the quotient of an object of $\operatorname{Mon}(L)$.

An embedding that satisfies the first two conditions is called a powerful embedding. This proposition is somewhat technical and the proof appears in the appendix. How ever, there is one part of the proof (of (iii)) that is central to a later argument. It is, in fact, the only part of the argument that uses the total connectedness of $L$.

LEMma 2. Let $X(a), a \neq 0$ be a family of sets indexed by the non-zero elements of $L$. Then there is a sheaf $X^{\sim}$ defined by $X^{\sim}(a)=\Sigma_{b \geq a} X(b)$ for $a \neq 0$ and $X^{\sim}(0)=1$.

PROOF. If $a_{1} \leq a_{2}$, then $\left\{b \mid b \geq a_{2}\right\} \subseteq\left\{b \mid b \geq a_{1}\right\}$ so that $X^{\sim}\left(a_{2}\right)$ is a summand of $X^{\sim}\left(a_{1}\right)$ and we let the restriction map be the inclusion. Then it is evident that we have a presheaf. To see that we have a sheaf, suppose $a=V a_{i}$ and $\left\{x_{i} \in S\left(a_{i}\right)\right\}$ are given as in the definition of sheaf. Then each $x_{i} \in X^{\sim}\left(b_{i}\right)$ for some $b_{i} \geq a_{i}$. But the fact that $x_{i}\left|a_{i} \wedge a_{j}=x_{j}\right| a_{i} \wedge a_{j}$ implies that $b_{i}=b_{j}$ and is not less than both $a_{i}$ and $a_{j}$. Thus $b_{i}=b$ is independent of $i$ and since $b \geq a_{i}$, for each $i, b \geq a_{i}$, whence $x=x_{i} \in S(a)$.

Theorem 3. Let $L$ be an arbitrary locale and let $T: \mathbf{F u z}(L) \rightarrow \operatorname{Mon}\left(L^{+}\right)$be defined by $T(X)=X^{\sim}$. Then $T$ determines a functor which is an equivalence of categories. 
Proof. We must describe the value of $T$ on morphisms. To do this, we let $f: X \rightarrow Y$ be a morphism of fuzzy sets. Then for any $x \in X^{\sim}(a)$, there is a unique $b \geq a$ such that $x \in X(b)$, whence $f(x) \in Y(c)$ for some $c \geq b$. But then $c \geq a$ so that $f(x) \in Y^{\sim}(a)$. Two things have to be verified that are trivial and are left to the reader. The first is that $T f$ is a morphism, that is that for $a \leq b$, and $x \in T X(b)$, $\operatorname{Tf}(x) \mid a=\operatorname{Tf}(x \mid a)$. The second is that $T$ is a functor, that is that for any $f: X \rightarrow$ $Y$ and $g: Y \rightarrow Z, T(g \circ f)=T g \circ T f$.

This defines $T f$. It is evident that $f \neq g$ implies that $T f \neq T g$ so that $T$ is faithful. Next let $g: T X \rightarrow T Y$. Let $x \in T X(a)$. Then there is a unique $b \geq a$ for which $x \in X(b)$. We have $g(b)(x) \in T Y(b)$ which means that there is a unique $c \geq b$ such that $g(b)(x) \in Y(c)$. Then let $f: X \rightarrow Y$ be defined by letting $f(x)$ be that element of $Y(c)$ which is $g(b)(x)$. Since $c \geq b, f: X \rightarrow Y$ is a morphism in $\mathbf{F u z}(L)$. That $T f=g$ is left to the reader.

The last thing to be shown is that every object of $\operatorname{Mon}(L)$ is isomorphic to one in the image of $T$. So let $S$ be an object of Mon $(L)$. We claim that for each element $x \in S(a)$, there is a unique $b \geq a$ such that there is an element $y \in S(b)$ with $y \mid a=x$ and that $y$ is unique. For consider the set of all $b_{i}$ for which there is such an element $y_{i}$. Since

$$
\left(y_{i} \mid b_{i} \wedge b_{j}\right)\left|a=y_{i}\right| a=x=y_{j}\left|a=\left(y_{j} \mid b_{i} \wedge b_{j}\right)\right| a
$$

and the restrictions are monic, it follows that $y_{i}\left|b_{i} \wedge b_{j}=y_{j}\right| b_{i} \wedge b_{j}$. The sheaf condition implies that if $b=V b_{i}$, then there is a $y \in S(b)$ with $y \mid b_{i}=y_{i}$. The uniqueness of $y$ follows from the fact that the restrictions are monic. Now let $S[a]$ denote the set of all elements of $S(a)$ which are not in the image of $S(b)$ for any $b>a$. It is clear that $S(a) \cong \sum_{b \geq a} S[b]$ so that $S=T X$ where $X(a)=S[a]$.

4. Regular and effectively regular categories. A category is called regular if it has finite limits and if the pullback of a regular epimorphism is a regular epimorphism. It is called effectively regular (called exact in [1]) if every equivalence relation is the kernel pair of some mapping. These are two notions which are important in the study of various kinds of categories. However, it would require a long detour to give a full discussion of these ideas here. I refer to either [1] or to [2] for a thorough treatment.

A functor between regular categories is called a regular functor if it preserves finite limits (for which finite products and equalizers are sufficient) as well as regular epimorphisms.

THEOREM 4. (Joyal, unpublished). For every regular category $R$ there is an effectively regular category $R^{\sim}$ and a regular embedding $R \rightarrow R^{\sim}$ which is universal for functors from $R$ into regular categories.

We will not prove this theorem since we do not need it. However, it can be proved by following the suggestions outlined in the discussion of how to make fuzzy sets fuzzier. In fact, we use the following theorem. 
THEOREM 5. Suppose $F: \mathscr{B} \rightarrow \mathscr{C}$ is a regular functor between regular categories. Suppose the following conditions are satisfied:

(i) $F$ is full and faithful.

(ii) Every subobject of an object of the form $F B$ is of the form $F B_{0}$ for some $B_{0} \subseteq B$.

(iii) Every object of $C$ is a quotient of an object of the form $F B$.

Then $F: \mathscr{B} \rightarrow \mathscr{C}$ is the effective completion of $\mathscr{B}$.

The proof will appear in the appendix.

Putting together our previous results, we conclude.

THEOREM 6. The effective completion of $\mathbf{F u z}(L)$ is the category $\mathbf{S h}\left(L^{+}\right)$.

Suppose we define $\mathbf{F u z}_{0}(L)$ to be the full subcategory of $\mathbf{F u z}(L)$ consisting of all the fuzzy sets $X$ for which $X(0)=\emptyset$. By using exactly the same arguments, one can readily show that if $L$ is totally connected, then $\mathbf{F u z}_{0}(L)$ is equivalent to the category $\operatorname{Mon}(L)$ and that its effective completion is $\mathbf{S h}(L)$. The conclusion is clear: when fuzzy sets are fuzzified further to allow fuzzy equality as well as fuzzy membership, the theory coalesces with topos theory. The entire structure of topos theory becomes available. For example, for each sheaf $S$, there is a sheaf $\mathbb{P} S$ interpreted as the power sheaf of $S$, so that morphisms $S^{\prime} \rightarrow \mathbb{P} S$ are in $1-1$ correspondence with subsets on $S \times S^{\prime}$, i.e. relations are from $S^{\prime}$ to $S$. This, in turn, allows all of intuitionistic set theory to be mirrored in these categories.

Despite this, some of the constructions of sheaf theory are available in $\mathbf{F u z}(L)$. For example, the existence of function sets has already been shown in [5]. The simple explanation is that when $X$ is an object of $\operatorname{Mon}(L)$, so is the exponential object $X^{Y}$ for any sheaf $Y$, a fact that is essentially trivial. In a similar way, one can demonstrate the existence of interpretations for first order logical constructions like $\exists$ and $\forall$ can also be demonstrated to exist in $\mathbf{F u z}(L)$. Again, it is trivial to see that this follows from the fact that the embedding is powerful, so that these operations on subobjects are inherited. However, $\mathbb{P} X$ will not generally belong to Mon $(L)$ when $X$ does, which explains why Mon $(L)$ does not have power objects. A different (or perhaps not so different) explanation appears in the introduction to this paper.

It is not hard to see that the categories of the form $\mathbf{S h}(L)$ for $L$ a locale are characterized by the fact that the subobjects of 1 are a set of generators. Although Gouguen claims in his 1974 paper that his axioms are better than the axioms for ordinary set theory, being categorical (in the logical sense), this claim is actually empty since his axiom of completeness relativizes the categoricity of his axioms to that of the underlying (ordinary) set theory.

The interested reader will find a rich field of intuitionistic analysis by exploring the topos theoretic literature (begin with [2], [4], [6] and [7] and follow the bibliographical pointers there). Questions such as what object is the object of reals (each possible definition - by cuts, by Cauchy sequences, by approximate homomorphisms on the natural numbers - will give its own concept of real numbers) have been extensively explored. 
Appendix. In this appendix, we have put two proofs of a more technical nature which can be read by anyone with some knowledge of category theory, particularly of regular categories.

Proof OF Proposition 1. Since the restriction of a mono is a mono and the product of a mono, each of the first two properties follows immediately once we know that both monomorphisms and products are computed "pointwise", that is if $f: S \rightarrow S^{\prime}$ is a mono iff $f(a)$ is injective for every $a \in L$ and that when $S=\Pi S_{i}$, then $S\left(a=\Pi S_{i}(a)\right.$ for all $a \in L$. But both of these properties are standard properties of sheaf categories and the proofs are found in any reference on topos theory, for example [2]. The relevant references are Exercise LIMFUN, page 47 for the proof in presheaf categories, Corollary 2 on page 54 for the proof that a left adjoint functor preserves limits, Theorem 9 on page 211 for the proof that the inclusion of sheaves into presheaves has a left adjoint and Exercise PBM on page 34 to see why a left exact functor preserves monos. As for the third assertion, let $X(a)=S(a)$ for $0 \neq a \in L$ and apply Lemma 2 .

Proof of Proposition 5. We must show that any regular functor if $G: \mathscr{B} \rightarrow \mathscr{E}$ into a category $\mathscr{E}$ which is effectively regular has a unique extension to a regular functor $G^{\sim}: \mathscr{C} \rightarrow \mathscr{E}$. Without loss of generality we may suppose that $\mathscr{B}$ is a full subcategory of $\mathscr{C}$ and that $F$ is the inclusion functor. So let $C$ be an object of $\mathscr{C}$ and choose a regular epimorphism $B_{0} \rightarrow C$. Since $B_{1}=B_{0} \times{ }_{C} B_{0}$ is a subobject of $B_{0} \times B_{0}$, it belongs to $\mathscr{B}$ and so we have a resolution

$$
B_{1} \rightrightarrows B_{0} \rightarrow C
$$

with both $B_{0}$ and $B_{1}$ in $\mathscr{B}$. We extend $G$ by letting

$$
G B_{1} \rightrightarrows G B_{0} \rightarrow G^{\sim} C
$$

be a coequalizer. We first show that this is independent of the resolution. If we have another resolution

$$
B_{1}^{\prime} \rightrightarrows B_{0}^{\prime} \rightarrow C
$$

build the diagram

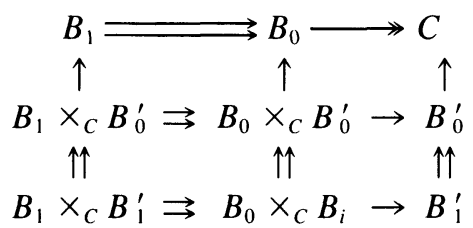

apply $G$ and use a standard diagram chase to show that the coequalizers of the upper row is the same as that of the left hand column. To extend the definition to morphisms, suppose we have a morphism $f: C \rightarrow C^{\prime}$. First choose a resolution

$$
B_{1} \rightrightarrows B_{0} \rightarrow C
$$


and a regular epi $B_{0}^{\prime} \rightarrow C^{\prime}$. By replacing $B_{0}^{\prime}$ by the pullback $B_{0}^{\prime} \times B_{0}$ which in a regular category still maps by a regular epimorphism to $C^{\prime}$, we can suppose that we have a commutative diagram

$$
\begin{gathered}
B_{1} \rightrightarrows B_{0} \longrightarrow C \\
\uparrow f_{1} \uparrow \uparrow f_{0} \uparrow \uparrow \\
B_{1}^{\prime} \rightrightarrows B_{0}^{\prime} \longrightarrow C^{\prime}
\end{gathered}
$$

where $B_{1}^{\prime}=B_{0}^{\prime} \times_{C^{\prime}} B_{0}^{\prime}$. Now apply $G^{\sim}$ to get the induced $G^{\sim} f$.

\section{REFERENCES}

1. M. Barr, Exact Categories, in Exact Categories and Categories of Sheaves, Lecture Notes in Mathematics, Vol. 236 Springer-Verlag (1972), pp. 1-120.

2. M. Barr and C. F. Wells, Triples, Toposes and Theories, Springer-Verlag, (1985).

3. M. Eytan, Fuzzy sets: A topos-logical point of view, Fuzzy Sets and Systems, 5 (1981), pp. 47-67.

4. M. P. Fourman and D. S. Scott, Applications of Sheaves, Lecture Notes in Mathematics, Vol. 753 Springer-Verlag, (1979).

5. J. Goguen, Concept representation in natural and artificial languages: Axioms, extensions and applications for fuzzy sets, Int. J. Man-Machine Studies, 6 (1974), pp. 513-561.

6. D. Higgs, A category approach to Boolean valued models, Preprint, University of Waterloo, (1973).

7. P. T. Johnstone, Stone Spaces, Cambridge Studies in Advanced Mathematics, Vol. 3 Cambridge University Press, (1982).

8. A. M. Pitts, Fuzzy sets do not form a topos, Fuzzy Sets and Systems, 8 (1982), pp. 101-104.

Department of Mathematics And Statistics

MCGILL UNIVERSITY

805 SHERbroOKe St., W.

Montréal, Québec

CANADA H3A 2K6 